\title{
THE PROPERTIES OF INFRARED GALAXIES IN THE LOCAL UNIVERSE
}

\author{
B. T. Soifer ANd G. Neugebauer \\ Division of Physics, Mathematics, and Astronomy, California Institute of Technology, Pasadena, California 91125 \\ Received 9 August 1990; revised 21 September 1990
}

\begin{abstract}
The $60 \mu \mathrm{m}$ selected IRAS Bright Galaxy Sample is used as a starting point to derive additional complete flux-limited samples of extragalactic objects at 12,25 , and $100 \mu \mathrm{m}$. With these complete samples the luminosity functions at all IRAS wavelengths are derived for the local Universe. These luminosity functions are used to determine the infrared emission of the local Universe. The maximum in the energy output of galaxies occurs at $100 \mu \mathrm{m}$. The infrared emission of galaxies at 12 and $25 \mu \mathrm{m}$ represents $\sim 30 \%$ of the total infrared luminosity in the local Universe. The mean infrared colors of infrared selected galaxies vary systematically with infrared luminosity; the ratio $S_{v}(60 \mu \mathrm{m}) / S_{v}(100 \mu \mathrm{m})$ increases and $S_{v}(12 \mu \mathrm{m}) / S_{v}(25 \mu \mathrm{m})$ decreases with increasing infrared luminosity.
\end{abstract}

\section{INTRODUCTION}

The IRAS all sky survey has provided a view of the infrared properties of a significant number of galaxies. Early in the study of infrared emission of galaxies it was realized that $60 \mu \mathrm{m}$ was the wavelength at which galaxies were detected most efficiently in the IRAS survey, and therefore one of the first aspects of the infrared sky to be studied was the luminosity function of galaxies selected in flux-limited samples at $60 \mu \mathrm{m}$. Several groups (Lawrence et al. 1986; Rowan-Robinson et al. 1987; Smith et al. 1987; Soifer et al. 1986, 1987, hereafter we refer to Soifer et al. 1987 as Paper I) studied the $60 \mu \mathrm{m}$ luminosity function of galaxies determined from samples of galaxies selected from the IRAS data based on different $60 \mu \mathrm{m}$ flux density limits; the derived luminosity functions all agree quite well. Recently Saunders et al. (1990) have compiled a summary luminosity function based on a number of these $60 \mu \mathrm{m}$ flux-limited samples, including most of those mentioned above. The luminosity functions were determined from samples that ranged over a factor of $\sim 10$ in $60 \mu \mathrm{m}$ flux density, and hence, over a factor of $\sim 3$ in distance. The agreement between the samples (see Paper I and Saunders et al. for comparisons of the various luminosity functions) implies that the overall density of the infrared bright galaxies is fairly uniform over the range of 30-100 $\operatorname{Mpc}\left(H_{0}=75 \mathrm{~km} \mathrm{~s}^{-1} \mathrm{Mpc}^{-1}\right)$, i.e., the density does not vary by more than a factor of $\sim 2$ over this range in distances.

In order to fully describe the global infrared emission from the local Universe, it is important to understand the infrared emission of galaxies throughout the infrared. This can be done in an unbiased manner by choosing complete flux-limited samples of extragalactic sources based on their emission at 12,25 , and $100 \mu \mathrm{m}$ as well as at $60 \mu \mathrm{m}$, i.e., based on their emission in the four IRAS wavelength bands.

The infrared emission from galaxies is thought to be predominantly thermal emission by dust (e.g., Soifer et al. 1987). Because the galaxies chosen to be bright at $60 \mu \mathrm{m}$ are dust rich, it is reasonable to expect that these galaxies will have dust with a range of temperatures, and hence will be bright at the other IRAS wavelengths. Indeed, the BGS does contain the brightest galaxies found at the other IRAS wavelengths. This correlation reflects the fact that the brightness of thermal emission at different temperatures is mainly determined by the quantity of dust which is present at these temperatures, and the galaxies that are dust rich in general have more dust emitting over the entire IRAS wavelength range. It is therefore possible to derive, from the BGS, subsamples chosen by their emission at the other IRAS wavelengths to be complete samples of galaxies at those wavelengths. Since samples chosen at the IRAS wavelengths span the range of dust temperatures that produce significant infrared emission in galaxies, these samples serve to describe the major infrared constituents of the Universe.

In this paper we derive such complete samples as subsamples of the IRAS Bright Galaxy Sample (BGS), and use these complete subsamples to derive the luminosity function in the local Universe at all the IRAS wavelengths. From these luminosity functions we then derive the energy distribution of the emergent infrared emission in the local Universe. We also examine the systematic change with luminosity in the infrared colors of the infrared selected galaxies.

\section{THE DATA}

The IRAS Bright Galaxy Sample (BGS, Paper I, Soifer $e t$ al. 1989, hereafter referred to as Paper II) can be used as a convenient dataset to extract subsamples that represent significant complete, flux-limited samples at the wavelengths other than $60 \mu \mathrm{m}$. The BGS represents a sample of the brightest galaxies found in the sky at $60 \mu \mathrm{m}$ and is complete to $5.24 \mathrm{Jy}$ at $60 \mu \mathrm{m}$. In Paper II, IRAS observations were reported at $12,25,60$, and $100 \mu \mathrm{m}$ for all the galaxies in the BGS. Nearly all the galaxies were detected at all IR $A S$ wavelengths as well as at $60 \mu \mathrm{m}$; out of 313 galaxies in the BGS, all were detected at $100 \mu \mathrm{m}, 312$ were detected at $25 \mu \mathrm{m}$, and 300 were detected at $12 \mu \mathrm{m}$. At high flux densities, all these subsamples show the same slope in the number versus flux density $\left(\log N\right.$ vs $\log S_{v}$ ) relation (see Fig. A1). At lower flux densities, the $\log N$ vs $\log S_{v}$ curves at 12,25 , and 100 $\mu \mathrm{m}$ flatten. This suggests that the subsamples are complete, flux density limited samples at the flux levels above the flattening, and are increasingly incomplete at lower flux densities. The flux density limit of the complete subsample of the BGS at $12 \mu \mathrm{m}$ is $0.79 \mathrm{Jy}$ and contains 122 sources, at $25 \mu \mathrm{m}$ the flux density limit of the complete subsample is $1.26 \mathrm{Jy}$ and contains 155 galaxies, while at $100 \mu \mathrm{m}$ the complete subsample contains 194 galaxies above a completeness limit of $15.85 \mathrm{Jy}$. The selection of these subsamples as well as comparison with other samples of IRAS selected galaxies is described in the Appendix. 


\section{DISCUSSION \\ 3.1 Distributions of Colors}

In Fig. 1 the color distributions of the BGS and the complete subsamples of the brightest objects selected at the other $I R A S$ wavelengths are displayed. The mean properties of the complete subsamples, including the entire BGS, are included in Table 1. The general effect is that the 12 and $25 \mu \mathrm{m}$ samples are warmer than the BGS, while the $100 \mu \mathrm{m}$ sample is cooler than the BGS at the selection wavelength.

The most extreme change in color distributions is seen for the $12 \mu \mathrm{m}$ subsample in Fig. 1(a), where the mean ratio of $S_{v}(12 \mu \mathrm{m}) / S_{v}(60 \mu \mathrm{m})$ increases by $40 \%$ from the complete BGS. In addition, while the 12 and $60 \mu \mathrm{m}$ subsample show the same limiting ratios of $S_{v}(12 \mu \mathrm{m}) / S_{v}(60 \mu \mathrm{m})$ at high ratios, the lowest values of $S_{v}(12 \mu \mathrm{m}) / S_{v}(60 \mu \mathrm{m})$ for the $12 \mu \mathrm{m}$ selected sample are almost an order of magnitude larger than found for the $60 \mu \mathrm{m}$ selected galaxies. The largest values of $S_{v}(12 \mu \mathrm{m}) / S_{v}(60 \mu \mathrm{m})$ observed in the BGS 12 $\mu \mathrm{m}$ subsample are $\log S_{v}(12 \mu \mathrm{m}) / S_{v}(60 \mu \mathrm{m}) \sim-0.7$. Spinoglio \& Malkan (1989) have found that $12 \mu \mathrm{m}$ selected galaxies extend to $\log S_{v}(12 \mu \mathrm{m}) / S_{v}(60 \mu \mathrm{m}) \sim 0$, but the galaxies with $\log S_{v}(12 \mu \mathrm{m}) / S_{v}(60 \mu \mathrm{m})>-0.7$ represent a small fraction, $<5 \%$, of $12 \mu \mathrm{m}$ selected galaxies.

The histograms of the ratio of $S_{v}(25 \mu \mathrm{m}) / S_{v}(60 \mu \mathrm{m})$ for the complete $25 \mu \mathrm{m}$ subsample and BGS are shown in Fig. 1(b). Here the mean value of the flux density ratio increases by $17 \%$ from the BGS to the $25 \mu \mathrm{m}$ selected subsample. The distributions of $S_{v}(25 \mu \mathrm{m}) / S_{v}(60 \mu \mathrm{m})$ ratios is not dramatically different for the two samples but shows clearly the larger values of this ratio found in the $25 \mu \mathrm{m}$ selected subsample.

Figure 1(c) shows the histogram of ratios of $S_{v}(60 \mu \mathrm{m}) / S_{v}(100 \mu \mathrm{m})$ for the BGS and complete 100 $\mu \mathrm{m}$ subsample; the mean ratio is $5 \%$ larger for the $100 \mu \mathrm{m}$ subsample compared to the BGS. The distributions of the flux density ratio are quite similar in the two samples, consistent with the small change in mean ratio, and show that the distribution of $S_{v}(60 \mu \mathrm{m}) / S_{v}(100 \mu \mathrm{m})$ ratios for galaxies chosen at $100 \mu \mathrm{m}$ is not significantly different from that found for the $60 \mu \mathrm{m}$ selected galaxies.

The histograms of Fig. 1 confirm the observation that many of the infrared properties of galaxies are well described by samples chosen at $60 \mu \mathrm{m}$, and those galaxies that show extreme colors in the $I R A S$ wavelengths are a small fraction of the galaxies in the local Universe (see the Appendix for more discussion of this point).

\subsection{Luminosities}

While the mean distances of the subsamples represent a complex function of the completeness limit of the samples and the energy distributions of galaxies, the distributions of total luminosities are a discriminator between the intrinsic properties of the different wavelength selected galaxy subsamples. In Fig. 2 histograms of the total $8-1000 \mu \mathrm{m}$ infrared luminosities of the galaxies in each subsample are plotted, normalized to the same total number for each subsample. The luminosity is based on the total measured flux from 8 to $120 \mu \mathrm{m}$, with a small $(40 \%)$ correction applied for the flux longward of $120 \mu \mathrm{m}$ (Perault et al. 1990). The mean luminosities of the subsamples are given in Table 1 as well. In plotting these luminosities all galaxies estimated to be associated with the Virgo cluster, i.e., those within $4 \mathrm{Mpc}$ of the center of Virgo, based on the Virgocentric flow model of
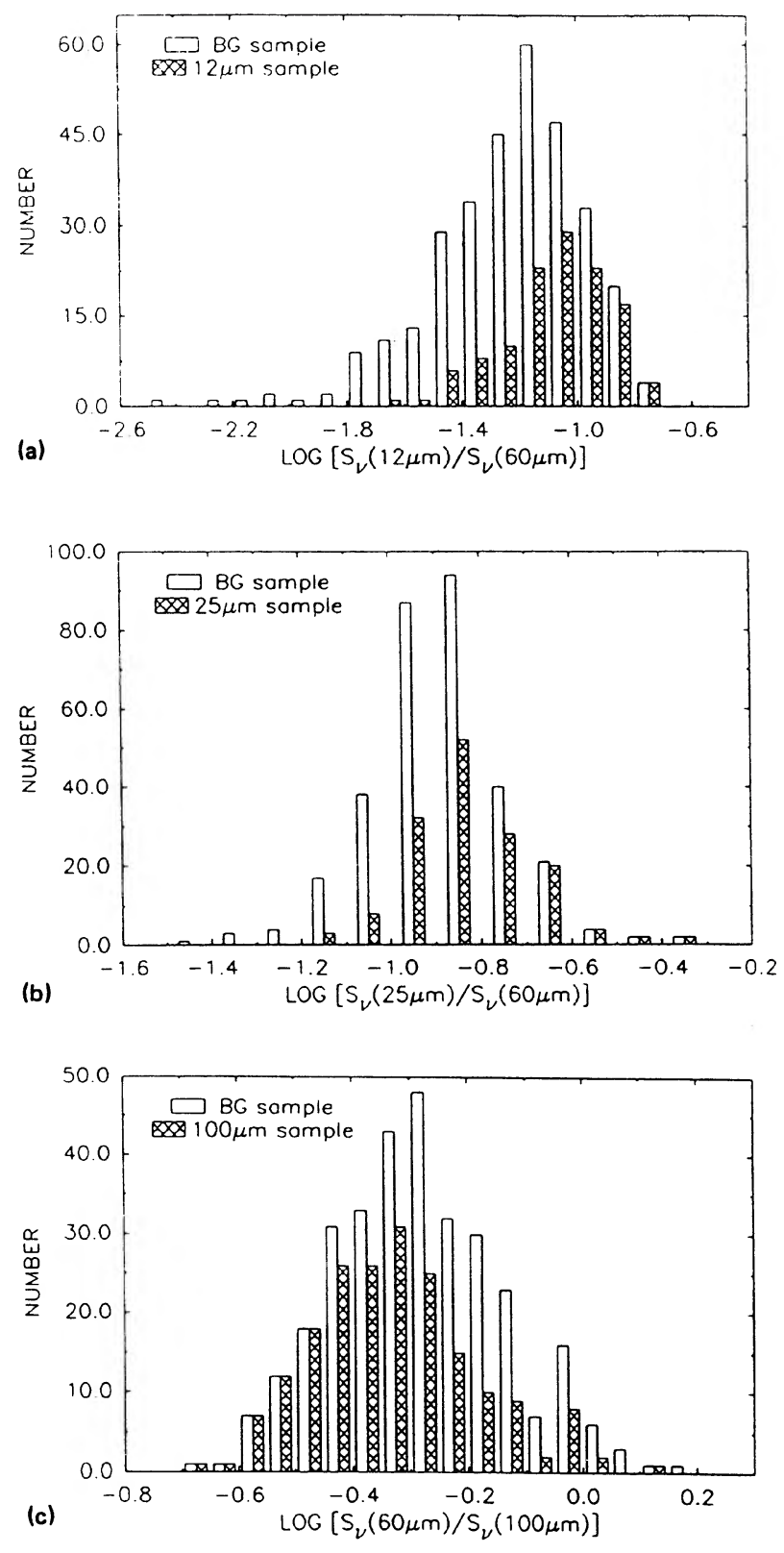

FIG. 1(a). Histograms of the number of galaxies in the complete Bright Galaxy Sample (open bars) and the $12 \mu \mathrm{m}$ subsample (hatched bars) with observed ratio of $\log S_{v}(12 \mu \mathrm{m}) / S_{v}(60 \mu \mathrm{m})$. The mean values of the ratio of $S_{v}(12 \mu \mathrm{m}) / S_{v}(60 \mu \mathrm{m})$ for the BGS and the $12 \mu \mathrm{m}$ subsample are given in Table 1. (b) Same as for (a) comparing the numbers of galaxies with observed ratio of $S_{v}(25 \mu \mathrm{m}) / S_{v}(60 \mu \mathrm{m})$ in the BGS and the $25 \mu \mathrm{m}$ subsample. (c) Same as for (a), comparing the numbers of galaxies with observed ratio of $S_{v}(60 \mu \mathrm{m}) / S_{v}(100 \mu \mathrm{m})$ in the BGS and the $100 \mu \mathrm{m}$ subsample.

Aaronson et al. (1982), with the distance to Virgo taken as $17.6 \mathrm{Mpc}$, have been excluded. Roughly $10 \%$ of the galaxies in each flux density limited subsample were judged, on this basis, to be associated with the Virgo cluster.

The distributions of the luminosities show no dramatic systematic effects, although the 25 and $60 \mu \mathrm{m}$ selected samples show larger fractions of galaxies with $L_{\mathrm{IR}}>10^{11} L_{\odot}$. 
TABLE 1. Properties of complete samples.

\begin{tabular}{|c|c|c|c|c|c|c|}
\hline $\begin{array}{l}\text { Wavelength } \\
\text { subsample }\end{array}$ & $\begin{array}{c}\text { Flux density } \\
\text { limit } \\
{[\mathrm{Jy}]}\end{array}$ & Number & $\begin{array}{c}\langle\text { Distance }\rangle \\
{[\mathrm{Mpc}]}\end{array}$ & $\left\langle\log \left(L_{\mathrm{IR}} / L_{\odot}\right)\right\rangle$ & $\left\langle\log S_{v}(\lambda) / S_{v}(60 \mu \mathrm{m})\right\rangle$ & Notes \\
\hline $12 \mu \mathrm{m}$ & 0.79 & $\begin{array}{l}104 \\
122\end{array}$ & $\begin{array}{l}17.5 \\
17.3\end{array}$ & 10.38 & $\begin{array}{l}-1.08 \\
-1.23\end{array}$ & $\begin{array}{l}(1) \\
(2) \\
(3)\end{array}$ \\
\hline $25 \mu \mathrm{m}$ & 1.26 & $\begin{array}{l}135 \\
151\end{array}$ & $\begin{array}{l}25.3 \\
24.1\end{array}$ & 10.62 & $\begin{array}{l}-0.82 \\
-0.90\end{array}$ & $\begin{array}{l}\text { (1) } \\
(2) \\
(3)\end{array}$ \\
\hline $60 \mu \mathrm{m}$ & 5.24 & $\begin{array}{l}282 \\
313\end{array}$ & $\begin{array}{l}33.0 \\
30.8\end{array}$ & 10.63 & & $\begin{array}{l}\text { (4) } \\
\text { (3) }\end{array}$ \\
\hline $100 \mu \mathrm{m}$ & 15.85 & $\begin{array}{l}172 \\
194\end{array}$ & $\begin{array}{l}22.5 \\
21.7\end{array}$ & 10.46 & $\begin{array}{l}0.28 \\
0.26\end{array}$ & $\begin{array}{l}\text { (1) } \\
(2) \\
(3)\end{array}$ \\
\hline
\end{tabular}

Notes to TABLE 1

(1) Subsample with Virgo galaxies excluded.

(2) Subsample with all galaxies included.

(3) BGS sample with all galaxies included.

(4) BGS sample with Virgo galaxies excluded.

The mean luminosities of the 25 and $60 \mu \mathrm{m}$ samples are indistinguishable, consistent with the finding from Paper II that the infrared luminosity does not depend on the 25-60 $\mu \mathrm{m}$ flux density ratio. On the other hand, the mean luminosities of the 12 and $100 \mu \mathrm{m}$ samples are $30 \%-50 \%$ lower than those of the 25 and $60 \mu \mathrm{m}$ samples. These results are also consistent with the findings of Papers I and II, where it was found that the mean $S_{v}(60 \mu \mathrm{m}) / S_{v}(100 \mu \mathrm{m})$ increases with infrared luminosity, and that the mean $S_{v}(12 \mu \mathrm{m}) / S_{v}(60 \mu \mathrm{m})$ decreases with luminosity. Since the 12 and $100 \mu \mathrm{m}$ selected samples automatically select galaxies with larger values of $S_{v}(12 \mu \mathrm{m}) / S_{v}(60 \mu \mathrm{m})$ and smaller values of $S_{v}(60 \mu \mathrm{m}) / S_{v}(100 \mu \mathrm{m})$, respectively, than does the $60 \mu \mathrm{m}$ selected sample, it is not surprising that these changes in the mean luminosity of the subsamples should occur.

\subsection{Luminosity Functions}

The luminosity functions for the complete subsamples were derived using the $V / V_{\mathrm{m}}$ technique (Schmidt 1968; Fel-

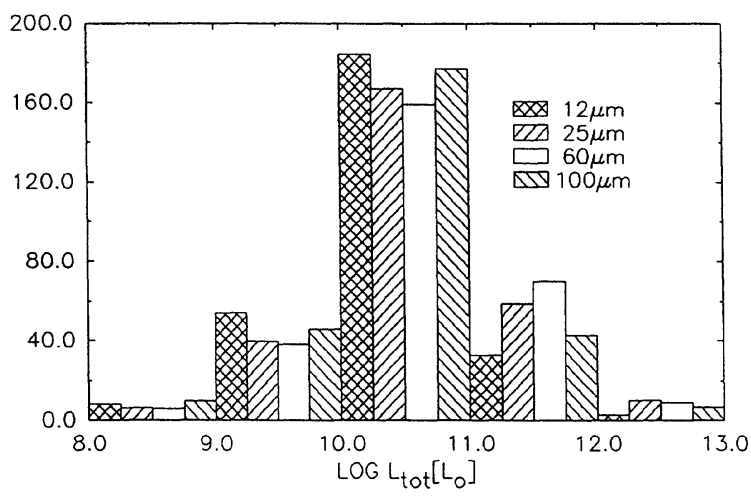

FIG. 2. Histograms of the distributions of $L_{\mathrm{IR}}$, the total 8-1000 $\mu$ m luminosities in solar bolometric luminosities for the BGS and the 12,25 , and $100 \mu \mathrm{m}$ subsamples. The histograms have been normalized to the same total area for each sample, and are plotted in luminosity bins of $10^{8}-10^{9} L_{\odot}, 10^{9}-10^{10} L_{\odot}$, etc. The mean values of $\log L$ for each sample are given in Table 1 . The 25 and $60 \mu \mathrm{m}$ (BGS) samples show significantly larger numbers of galaxies at $L>10^{11} L_{\odot}$ than do the 12 and $100 \mu \mathrm{m}$ samples. ton 1977) as described in Paper I. The solid angle covered by each sample is 14500 square deg, and the flux density limits for the samples are given in Table 1 . The $K$ corrections are based on power-law slopes measured for the galaxies using the $I R A S$ data. For the 12 and $25 \mu \mathrm{m}$ luminosity functions the slope is that determined between 12 and $25 \mu \mathrm{m}$, while at $100 \mu \mathrm{m}$ the slope for the $K$ correction is that determined from observations at 60 and $100 \mu \mathrm{m}$. Because the redshifts in all cases are small (the maximum redshift $z$ for the BGS is $z=0.08$ and $\langle z\rangle=0.006$ ), the $K$ corrections are also small.

The luminosity functions at 12,25 , and $100 \mu \mathrm{m}$ for the subsamples, along with the $60 \mu \mathrm{m}$ luminosity function, are plotted in Fig. 3. In all cases the abscissa is the luminosity per octave at the wavelength $\lambda, v L_{v}(\lambda)$, where $v$ is the frequency and $L_{v}$ is the luminosity density; the luminosity is plotted in units of solar bolometric luminosities $\left(3.83 \times 10^{26} \mathrm{~W}\right)$. Here those galaxies believed to be in the Virgo cluster (based on angular distance from the center of the Virgo cluster and radial velocity, see above) have been excluded, although no attempt was made to account for the small volume of space excluded from the determination of $V_{\mathrm{m}}$ by this technique $(<8 \%$, see Paper I).

As described in Paper I, the $60 \mu \mathrm{m}$ luminosity function

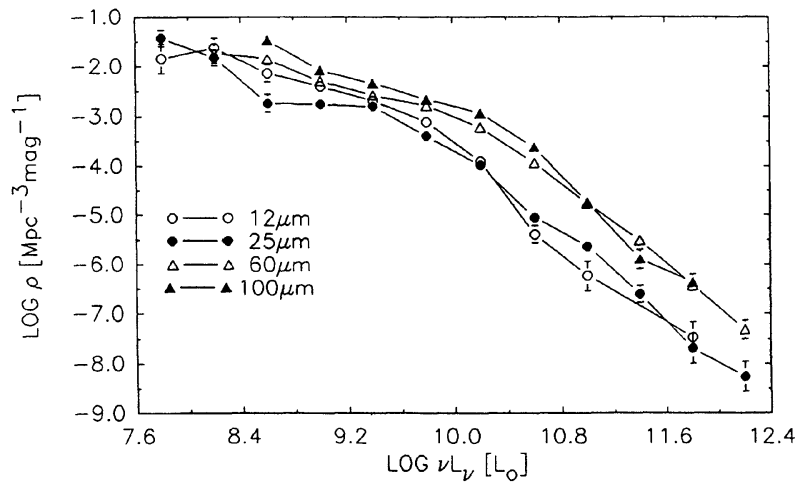

FIG. 3. The luminosity functions, plotted as space density vs luminosity for the $12,25,60 \mu \mathrm{m}$ (BGS), and $100 \mu \mathrm{m}$ samples. The luminosity is $v L_{v}(\lambda)$, in units of solar bolometric luminosities. 
derived for the BGS is in excellent agreement with those derived by others using different samples (e.g., Rowan-Robinson et al. 1987; Smith et al. 1987; Rieke \& Lebofsky 1986) and the summary $60 \mu \mathrm{m}$ luminosity function of Saunders $e t$ al. (1990). Saunders et al. find that the mean density of galaxies, averaged over nine samples, is about $30 \%$ lower that that found for the BGS alone. This is likely associated with the overdensity of the local supercluster. Since the BGS is the $60 \mu \mathrm{m}$ sample with the highest flux density limit of those considered by Saunders et al. it should be most affected by the local supercluster. The luminosity functions at 25 and $100 \mu \mathrm{m}$ derived here are in excellent agreement with the 25 and $100 \mu \mathrm{m}$ luminosity functions derived by Rowan-Robinson et al.

The $12 \mu \mathrm{m}$ luminosity function derived here disagrees substantially with the results presented by Spinoglio \& Malkan (1989, hereafter referred to as SM) in that the space density presented is on average a factor of $\sim 5$ greater than that of SM at the same luminosity. As will be derived later, the luminosity output of the local Universe at $12 \mu \mathrm{m}$ is consistent with the observed energy distributions of galaxies, and would be much different if the luminosity function of SM were valid. This gives us confidence that our $12 \mu \mathrm{m}$ luminosity function is valid.

It is difficult to understand the cause of the disagreement between the present $12 \mu \mathrm{m}$ luminosity function and that of $\mathrm{SM}$, since the overlap between the two datasets is excellent. The $12 \mu \mathrm{m}$ BGS subsample of 122 galaxies used has 15 galaxies not listed in SM, but this does not account for a factor of 5 discrepancy in space densities. One possible source of the discrepancy could be a disagreement on the completeness limit of their sample. The discrepancy might result if the true completeness limit of SM corresponded to larger flux densities than assumed by SM.

The space density of galaxies at a given luminosity per octave is greater at $100 \mu \mathrm{m}$ than at $60 \mu \mathrm{m}$. This is not surprising based on the relative $60-100 \mu \mathrm{m}$ flux densities of the BGS and the $100 \mu \mathrm{m}$ subsample. From Fig. 1 the vast majority of the galaxies have $\log S_{v}(60 \mu \mathrm{m}) / S_{v}(100 \mu \mathrm{m})<0.22$, i.e., have more energy, $v L_{v}$, emerging at $100 \mu \mathrm{m}$ than at $60 \mu \mathrm{m}$. What is more surprising is the substantial contribution to the emergent luminosity of galaxies at 12 and $25 \mu \mathrm{m}$. At lower luminosities the space density of galaxies emitting a fixed luminosity at $12 \mu \mathrm{m}$ is within a factor of 3 of that in the far infrared, while the space density decreases significantly compared to the space density at $60 \mu \mathrm{m}$ for galaxies at $v L_{v}>10^{10.5} L_{\odot}$. Presumably this drop in the contribution to the infrared luminosity at $12 \mu \mathrm{m}$ is a reflection of the decreasing luminosity at $12 \mu \mathrm{m}$ compared to total luminosity in high luminosity systems. The $25 \mu \mathrm{m}$ luminosity function of galaxies appears to be a constant fraction, $1 / 10$ of that at $60 \mu \mathrm{m}$, again reflecting the lack of systematic change of the $S_{v}(25 \mu \mathrm{m}) / S_{v}(60 \mu \mathrm{m})$ ratio with galaxy luminosity.

\subsection{The Infrared Luminosity of the Local Universe}

The total infrared energy output of the local Universe has been estimated in two ways. One approach is to integrate each of the wavelength selected luminosity functions to derive a luminosity density at each wavelength, and integrate the resultant energy distribution. This energy distribution is shown in Fig. 4; the total infrared luminosity density from 8 to $120 \mu \mathrm{m}$ implied by this distribution is $9.5 \times 10^{7} L_{\odot} / \mathrm{Mpc}^{3}$. Applying a correction for the out-of-band flux beyond 120 $\mu \mathrm{m}$ (Perault et al. 1990) leads to an estimate of the total

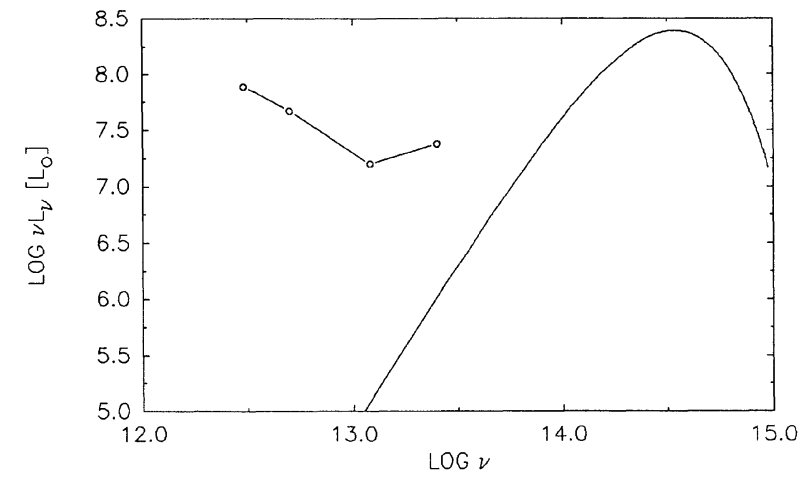

FIG. 4. The luminosity density of the local Universe, plotted as luminosity per octave $\left(v L_{v}\right)$ in $L_{\odot}$ per Mpc from the visible through $100 \mu \mathrm{m}$. The data points at $12-100 \mu \mathrm{m}$ (open circles) are derived by integrating the observed luminosity functions (from Fig. 3), and are given in Table 3. The curve is a blackbody of temperature $4200 \mathrm{~K}$, which is a crude approximation to the mean temperature of galaxy light (Aaronson 1977) between 0.5 and 2.2 $\mu \mathrm{m}$. The normalization of the blackbody curve is based on the estimated bolometric contribution of stellar photospheres to the total luminosity output of the local Universe as given in Paper I.

infrared luminosity from 8 to $1000 \mu \mathrm{m}$ of $1.24 \times 10^{8}$ $L_{\odot} / \mathrm{Mpc}^{3}$, or roughly $40 \%$ greater than the $40-1000 \mu \mathrm{m}$ luminosity density derived from the 60 and $100 \mu \mathrm{m}$ fluxes of the BGS in Paper I.

The correction for out-of-band luminosity assumes the temperature for the dust is well defined by the IRAS 60 and $100 \mu \mathrm{m}$ flux densities. Some recent studies, e.g., Devereux \& Young (1990) have argued that there is a significant quantity of cold dust in many of these galaxies. Even if this suggestion is correct, the contribution of cold dust to the infrared luminosity of the local universe is comparatively small, since the material is quite cold $(<20 \mathrm{~K})$ so that the luminosity of the cold dust is much less than that of the dust detected by IRAS.

A second approach to estimating the bolometric infrared output of galaxies is to derive the bolometric luminosity function using a bolometric flux-limited sample, and to integrate this luminosity function. Consequently, a complete bolometric flux-limited subsample was derived from the BGS, based on the integrated flux $F$ of each galaxy from 8 to 120 $\mu \mathrm{m}$ with a completeness limit, determined from the $\log N$ vs $\log F$ plot for the BGS, of $5 \times 10^{-13} \mathrm{~W} / \mathrm{m}^{2}$. Using this sample, and integrating the bolometric luminosity function over the observed range gives a total energy output of $1.1 \times 10^{8}$ $L_{\odot} / \mathrm{Mpc}^{3}$, in excellent agreement with that derived from the wavelength selected luminosity functions.

The luminosity density from 8 to $40 \mu \mathrm{m}$ is $\sim 40 \%$ of that emitted longwards of $40 \mu \mathrm{m}$. It is generally accepted that this emission is a result of the heating of small grains (PAHs) by single UV or optical photons (see, e.g., Puget \& Leger 1989, for a review). In early type galaxies a major fraction, if not the vast majority of the emission at $12 \mu \mathrm{m}$, comes from hot dust surrounding late-type stars (e.g., Jura et al. 1987; Soifer et al. 1986), however the $12 \mu \mathrm{m}$ luminosity of these galaxies does not contribute significantly, compared to late-type spirals, in the local universe. The relative energy outputs shortwards and longwards of $40 \mu \mathrm{m}$ measure the absorption cross sections of small grains, compared to the large grains of the "standard" model of interstellar dust. The small grains 
TABLE 2. Space densities of wavelength selected samples.

\begin{tabular}{|c|c|c|c|c|}
\hline $\begin{array}{c}\log \left(v L_{v}\right) \\
{\left[L_{\odot}\right]}\end{array}$ & $\begin{array}{c}12 \mu \mathrm{m} \\
\log \rho\end{array}$ & $\begin{array}{l}25 \mu \mathrm{m} \\
\log \rho\end{array}$ & $\begin{array}{l}60 \mu \mathrm{m} \\
\log \rho\end{array}$ & $\begin{array}{c}100 \mu \mathrm{m} \\
\log \rho\end{array}$ \\
\hline $\begin{array}{r}7.80 \\
8.20 \\
8.60 \\
9.00 \\
9.40 \\
9.80 \\
10.20 \\
10.60 \\
11.00 \\
11.40 \\
11.80 \\
12.20\end{array}$ & $\begin{array}{l}-1.84 \pm 0.30 \\
-1.62 \pm 0.19 \\
-2.13 \pm 0.17 \\
-2.40 \pm 0.13 \\
-2.69 \pm 0.08 \\
-3.11 \pm 0.07 \\
-3.91 \pm 0.10 \\
-5.39 \pm 0.18 \\
-6.24 \pm 0.30 \\
-7.47 \pm 0.30\end{array}$ & $\begin{array}{l}-1.43 \pm 0.16 \\
-1.82 \pm 0.16 \\
-2.74 \pm 0.18 \\
-2.76 \pm 0.11 \\
-2.81 \pm 0.07 \\
-3.40 \pm 0.07 \\
-3.99 \pm 0.07 \\
-5.05 \pm 0.11 \\
-5.64 \pm 0.11 \\
-6.61 \pm 0.17 \\
-7.70 \pm 0.30 \\
-8.26 \pm 0.30\end{array}$ & $\begin{array}{l}-1.74 \pm 0.20 \\
-1.85 \pm 0.15 \\
-2.29 \pm 0.13 \\
-2.58 \pm 0.09 \\
-2.79 \pm 0.06 \\
-3.23 \pm 0.05 \\
-3.94 \pm 0.06 \\
-4.78 \pm 0.08 \\
-5.52 \pm 0.09 \\
-6.43 \pm 0.13 \\
-7.32 \pm 0.18\end{array}$ & $\begin{array}{l}-1.47 \pm 0.15 \\
-2.08 \pm 0.15 \\
-2.34 \pm 0.11 \\
-2.66 \pm 0.07 \\
-2.94 \pm 0.06 \\
-3.62 \pm 0.07 \\
-4.75 \pm 0.11 \\
-5.90 \pm 0.20 \\
-6.37 \pm 0.17\end{array}$ \\
\hline
\end{tabular}

absorb $\sim 30 \%$ of the dust heating photons in the infrared bright galaxies, and therefore represent a major constituent of the interstellar medium in galaxies. While these grains have a substantial cross section for absorbing UV photons, if they are as small as has been inferred, they contribute at most a few percent to the total mass of dust in the interstellar medium because the ratio of cross section to volume is substantially larger for these grains than "normal" grains (cf. Allamandola et al. 1989).

While galaxies with $L_{\mathrm{IR}}>10^{11} L_{\odot}$ have attracted much interest and study, they produce a small fraction of the infrared luminosity in the local Universe. From the luminosity functions given in Table 2, galaxies with $v L_{v}>10^{11} L_{\odot}$ at 60 $\mu \mathrm{m}$ produce at most $6 \%$ (at $60 \mu \mathrm{m}$ ) of the infrared emission in the local Universe. Like highly luminous galaxies in the visible, such systems are quite rare among the population of galaxies as a whole.

In Fig. 4, the energy output of the local Universe, from the visible through $100 \mu \mathrm{m}$ is plotted. The luminosity per octave $v L_{v}$ at the IRAS wavelengths is that derived here, and given in Table 3. The visible output was taken to be represented by a $4200 \mathrm{~K}$ blackbody, corresponding to the mean $V-K$ colors of galaxies (Aaronson 1978) with total bolometric luminosity of $4 \times 10^{8} L_{\odot} / \mathrm{Mpc}^{3}$ as derived in Paper I. It can be seen directly here that the infrared luminosity is roughly $30 \%$ of that in normal starlight in the Universe, and well in excess of the stellar photospheric emission at all the IRAS wavelengths.

\subsection{The Variation of the Infrared Colors of Galaxies With Luminosity}

For comparison with the energy emitted at each IRAS wavelength, in Fig. 5 the observed energy distributions for the BGS are plotted, averaged in luminosity bins, and normalized to $60 \mu \mathrm{m}$. The mean colors of the galaxies $\left\langle\log \left[v S_{v}(\lambda) / v S_{v}(60 \mu \mathrm{m})\right]\right\rangle$ within the bins of $8-1000$ $\mu \mathrm{m}$ luminosity are given in Table 4 . The distribution of the local emergent energy is quite similar to that of the lower

TABLE 3. Energy output of local universe.

\begin{tabular}{cccc}
\hline \hline \multicolumn{4}{c}{$\log v L_{\nu}\left(L_{\odot}\right)$} \\
\hline $12 \mu \mathrm{m}$ & $25 \mu \mathrm{m}$ & $60 \mu \mathrm{m}$ & $100 \mu \mathrm{m}$ \\
7.38 & 7.20 & 7.67 & 7.89 \\
\hline \hline
\end{tabular}

luminosity galaxies, but in all cases the ratio of energy emitted at wavelength $\lambda$ to that at $60 \mu \mathrm{m}$ is larger than the mean for any luminosity range of galaxies. This also is not surprising, since the emitted energy at a given wavelength is dominated by those galaxies which are brightest at that wavelength. Figure 5 also illustrates clearly the systematic change in the infrared colors of galaxies with luminosity (e.g., Paper II). It is seen that the mean of $S_{v}(60 \mu \mathrm{m}) / S_{v}(100 \mu \mathrm{m})$ increases with luminosity, while the mean of $S_{v}(12 \mu \mathrm{m}) / S_{v}(60 \mu \mathrm{m})$ decreases with luminosity; there is no luminosity dependence of $S_{v}(25 \mu \mathrm{m}) / S_{v}(60 \mu \mathrm{m})$.

The systematic change in the mean colors of galaxies with infrared luminosity is shown more clearly in Fig. 6, where the mean of $S_{v}(12 \mu \mathrm{m}) / S_{v}(25 \mu \mathrm{m})$ is plotted versus $S_{v}(60 \mu \mathrm{m}) / S_{v}(100 \mu \mathrm{m})$ for galaxies within the different luminosity bins. These data are given in Table 5 . This colorcolor plot represents a monotonic sequence of increasing ratio of $S_{v}(60 \mu \mathrm{m}) / S_{v}(100 \mu \mathrm{m})$ and decreasing ratio of $S_{v}(12 \mu \mathrm{m}) / S_{v}(25 \mu \mathrm{m})$ with increasing infrared luminosity.

Figures 5 and 6 show the decreasing importance of the small grain component of interstellar dust at higher luminos-

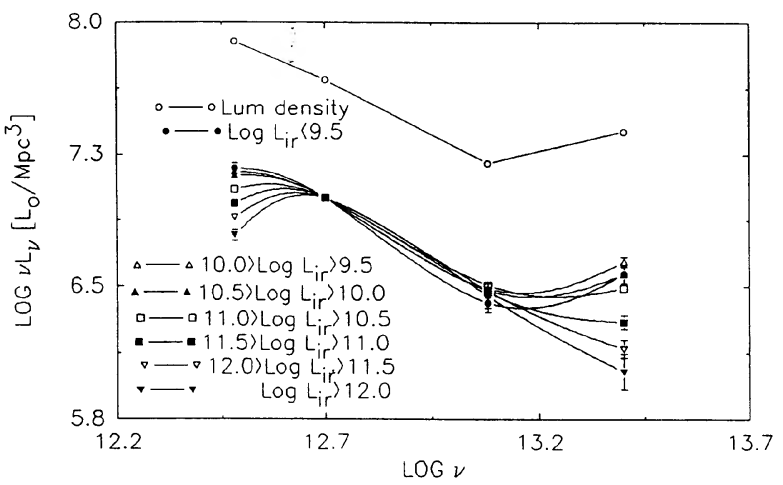

FIG. 5. The luminosity density of the local Universe, plotted as luminosity per octave, $v L_{v}$, in $L_{\odot} \mathrm{Mpc}^{-3}$ from 12 to $100 \mu \mathrm{m}$ (open circles). The data are the same as in Fig. 4. The lower curves are the mean energy distributions of galaxies in the BGS in luminosity bins. The energy distributions were determined by averaging $\log S_{v}(\lambda) / S_{v}(60 \mu \mathrm{m})$ for all the galaxies within the appropriate range of $L_{\mathrm{IR}}$, and plotting the mean colors, normalized to $\log v L_{v}(60 \mu \mathrm{m})=7.0$. The mean colors of galaxies in the luminosity bins are given in Table 4. 
TABLE 4. Mean infrared colors of galaxies.

\begin{tabular}{cccr}
\hline \hline \multicolumn{4}{c}{$\left\langle\log v L_{v}(\lambda) / v L_{v}(60 \mu \mathrm{m})\right\rangle$} \\
\hline $\begin{array}{c}\text { log } L_{\text {tot }} \\
{\left[L_{\odot}\right]}\end{array}$ & $12 \mu \mathrm{m}$ & $25 \mu \mathrm{m}$ & $100 \mu \mathrm{m}$ \\
\hline$<9.5$ & $-0.43 \pm 0.05$ & $-0.59 \pm 0.05$ & $0.17 \pm 0.02$ \\
$9.5-10.0$ & $-0.36 \pm 0.03$ & $-0.51 \pm 0.02$ & $0.15 \pm 0.02$ \\
$10.0-10.5$ & $-0.43 \pm 0.02$ & $-0.52 \pm 0.01$ & $0.13 \pm 0.01$ \\
$10.5-11.0$ & $-0.51 \pm 0.02$ & $-0.49 \pm 0.01$ & $0.05 \pm 0.01$ \\
$11.0-11.5$ & $-0.70 \pm 0.04$ & $-0.53 \pm 0.03$ & $-0.03 \pm 0.02$ \\
$11.5-12.0$ & $-0.85 \pm 0.05$ & $-0.52 \pm 0.04$ & $-0.11 \pm 0.02$ \\
$>12.0$ & $-0.98 \pm 0.10$ & $-0.55 \pm 0.07$ & $-0.21 \pm 0.03$ \\
\hline \hline
\end{tabular}

ities, particularly for $L_{\mathrm{IR}}>10^{10.5} L_{\odot}$. Boulanger et al. (1988) have suggested that these grains are increasingly depleted in the high radiation density environment of $\mathrm{H}$ iI regions and that exists in the more active galaxies. This depletion is likely due to the destruction of the small grains caused either by heating by higher energy photons or by absorption of multiple photons and multiple ionizations within a necessary short time interval (Puget 1990, private communication). Both these processes should proceed increasingly rapidly as the mean radiation energy density increases in galaxies.

$$
\text { 4. SUMMARY }
$$

The $60 \mu \mathrm{m}$ selected IRAS Bright Galaxy sample has been used to create subsamples of extragalactic sources that are complete, flux density limited samples at 12,25 , and $100 \mu \mathrm{m}$. Based on these complete samples the properties of $60 \mu \mathrm{m}$ selected galaxies have been compared with those selected at other IRAS wavelengths and the following properties of galaxies in the local Universe have been determined.

(i) The infrared luminosity from 8 to $1000 \mu \mathrm{m}$ of galaxies is $\sim 30 \%$ of that from the late-type stars that dominate the luminosity output of galaxies in the local Universe.

(ii) The infrared luminosity in the local Universe at wavelengths between 8 and $40 \mu \mathrm{m}$ is $\sim 40 \%$ of that at wavelengths $>40 \mu \mathrm{m}$. If, as conjectured, this emission is domi-

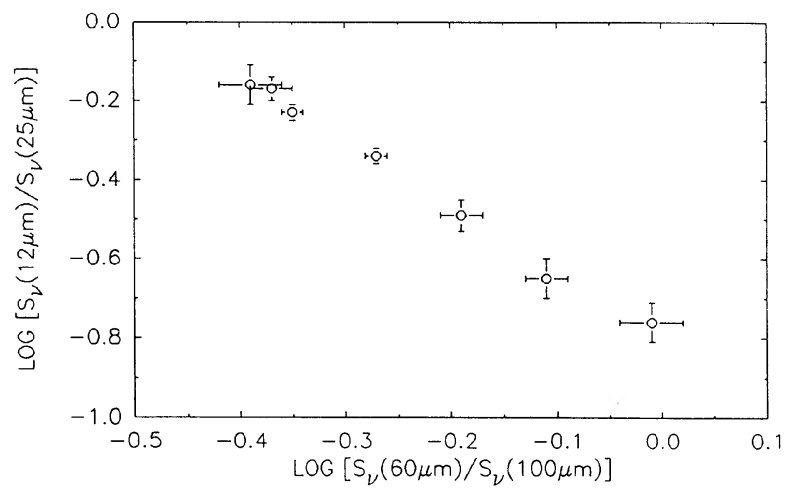

FIG. 6. The mean colors of galaxies, averaged in luminosity bins of $L_{\mathrm{IR}}$, in the BGS, plotted as $\log S_{v}(12 \mu \mathrm{m}) / S_{v}(25 \mu \mathrm{m})$ vs $\log S_{v}(60 \mu \mathrm{m}) /$ $S_{v}(100 \mu \mathrm{m})$. The colors are given in Table 5 . The mean colors change montonically from large values of $\log S_{v}(12 \mu \mathrm{m}) / S_{v}(25 \mu \mathrm{m})$ and small values of $\log S_{v}(60 \mu \mathrm{m}) / S_{v}(100 \mu \mathrm{m})$ at $L_{\mathrm{IR}}<10^{9.5} L_{\odot}$ to small values of $\log S_{v}(12 \mu \mathrm{m}) / S_{v}(25 \mu \mathrm{m})$ and large values of $\log S_{v}(60 \mu \mathrm{m}) /$ $S_{v}(100 \mu \mathrm{m})$ at $L_{\mathrm{IR}}>10^{12} L_{\odot}$.
TABLE 5. Mean colors of IRAS bright galaxies.

\begin{tabular}{ccc}
\hline \hline $\log L_{\mathrm{IR}}$ & $\left\langle\log S_{v}(12 \mu \mathrm{m}) /\right.$ & $\left\langle\log S_{v}(60 \mu \mathrm{m}) /\right.$ \\
{$\left[L_{\odot}\right]$} & $\left.\mathrm{S}_{v^{\prime}}(25 \mu \mathrm{m})\right\rangle$ & $-00 \mu \mathrm{m})\rangle$ \\
\hline$<9.5$ & $-0.16 \pm 0.05$ & $-0.39 \pm 0.03$ \\
$9.5-10.0$ & $-0.17 \pm 0.03$ & $-0.37 \pm 0.02$ \\
$10.0-10.5$ & $-0.23 \pm 0.02$ & $-0.35 \pm 0.01$ \\
$10.5-11.0$ & $-0.34 \pm 0.02$ & $-0.27 \pm 0.01$ \\
$11.0-11.5$ & $-0.49 \pm 0.04$ & $-0.19 \pm 0.02$ \\
$11.5-12.0$ & $-0.65 \pm 0.05$ & $-0.11 \pm 0.02$ \\
$>12.0$ & $-0.76 \pm 0.05$ & $-0.01 \pm 0.03$ \\
\hline \hline
\end{tabular}

nated by emission from small grains heated by single photons, then such small grains are a major component of the interstellar medium of galaxies. Such small grains must absorb a substantial fraction of the dust heating photons in galaxies, but carry only a few percent of the mass of dust because of their large absorption cross section to mass ratio.

(iii) The colors of infrared selected galaxies vary systematically with luminosity. The mean ratios of $S_{v}(12 \mu \mathrm{m}) / S_{v}(25 \mu \mathrm{m})$ and $S_{v}(12 \mu \mathrm{m}) / S_{v}(60 \mu \mathrm{m}) \mathrm{de}-$ crease, while the mean ratio of $S_{v}(60 \mu \mathrm{m}) / S_{v}(100 \mu \mathrm{m})$ increases as the 8-1000 $\mu \mathrm{m}$ luminosity of galaxies increases. We interpret this as a result of the fraction of small grains in the interstellar medium of galaxies decreasing as the luminosity increases.

Note added in proof. A significant correction, increasing all the space densities reported by SM by a factor of 2.5 , has been found by Malkan (1990, private communication). Coupled with the other small disagreements, this brings the luminosity functions into satisfactory agreement.

We thank D. B. Sanders, J. L. Puget, and M. Malkan for useful discussions. This work was supported in part by the NASA Astrophysics Data Program, and in part by the NASA Long Term Space Astrophysics Research Program.

\section{APPENDIX: USING THE IRAS BRIGHT GALAXIES TO DERIVE COMPLETE SAMPLES AT 12, 25, AND $100 \mu \mathrm{m}$}

To derive the luminosity functions at 12,25 , and $100 \mu \mathrm{m}$, it is necessary to begin with complete samples of extragalactic objects at each wavelength. The galaxies in the samples used were selected first by their brightness at $60 \mu \mathrm{m}$ and then by their brightness at another IRAS wavelength. The primary argument that these represent complete samples at the specific $I R A S$ wavelength is the similarity of the slope of the $\log N$ vs $\log S_{v}$ plot at these wavelengths to that at $60 \mu \mathrm{m}$ (above the completeness limit of the subsample). These plots, previously shown in Paper II, are repeated for convenience in Fig. A1. Because these samples contain the brightest galaxies at all the IRAS wavelengths, and have a similar $\log N-\log S_{v}$ slope to that found for the Bright Galaxy sample itself, we believe that these samples are complete above the flux level where the slopes flatten.

This approach represents a "shortcut" to selecting a complete sample at a given wavelength (other than $60 \mu \mathrm{m}$ ), one that potentially omits significant fractions of sources at each of the secondary wavelengths, i.e., those that are bright at the secondary wavelength but faint at the original selection wavelength of $60 \mu \mathrm{m}$. Because this is not based on a fluxlimited sample chosen directly at the specific wavelength, the approach must be tested for validity, to understand its limitations in deriving complete, flux-limited samples at wavelengths other than $60 \mu \mathrm{m}$. In particular, any systematic biases of this approach must be carefully understood. 

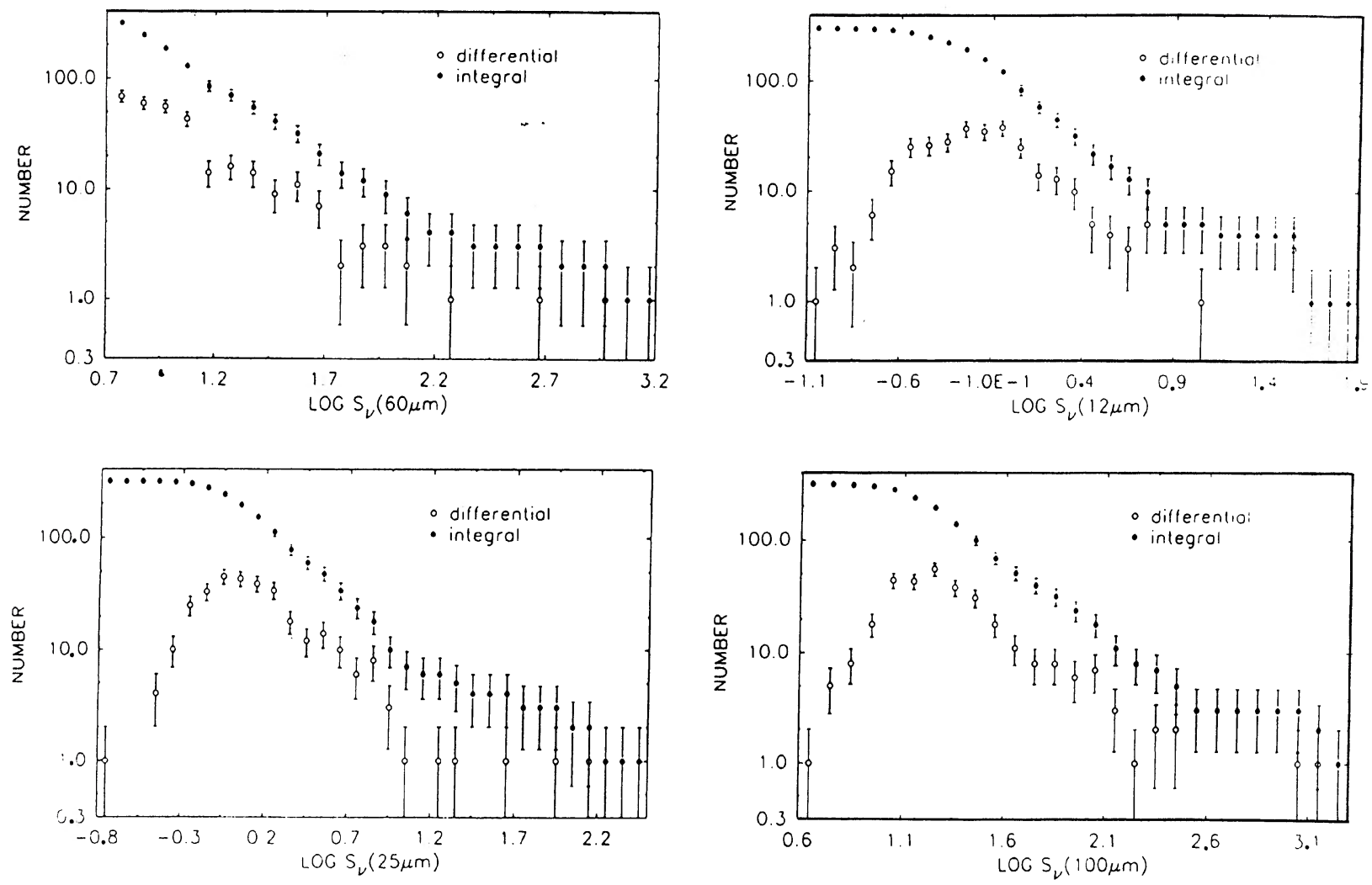

FIG. A1. The number vs flux-density relations plotted for the galaxies in the IRAS Bright Galaxy Sample. The data are for the 313 galaxies with $S_{v}(60 \mu \mathrm{m}) \geqslant 5.24 \mu \mathrm{m}$, and all panels show both integral $\left[N\left(S_{v}>S\right)\right]$ and differential $N\left[S_{v} \pm d(\log S)\right]$ counts. The bins have width $d(\log S)=0.1$. The errors shown are statistical errors only, i.e., $V N$. Only those galaxies with measured flux densities, i.e., 300 at $12 \mu \mathrm{m}, 312$ at $25 \mu \mathrm{m}$, and 313 galaxies at $100 \mu \mathrm{m}$ are included in the plots.

An estimate of the incompleteness of the BGS $12 \mu \mathrm{m}$ subsample comes from determining the fraction of galaxies in the $12 \mu \mathrm{m}$ selected sample of Spinoglio \& Malkan (1989, $\mathrm{SM})$ that have ratios of $S_{v}(12 \mu \mathrm{m}) / S_{v}(60 \mu \mathrm{m})$ greater than the greatest seen in the BGS $12 \mu \mathrm{m}$ subsample. Approximately 20 galaxies out of a total sample of 400 in the SM sample have $S_{v}(12 \mu \mathrm{m}) / S_{v}(60 \mu \mathrm{m})>0.2$, the largest value found in the BGS $12 \mu \mathrm{m}$ subsample (see below). This suggests that the incompleteness of the BGS $12 \mu \mathrm{m}$ subsample is $\sim 5 \%$, again an acceptably small fraction. It should be noted that $3 / 4$ of the objects in the SM sample with $S_{v}(12 \mu \mathrm{m}) / S_{v}(60 \mu \mathrm{m})>0.2$ are Seyfert 1 galaxies (more than half of the Seyfert 1 galaxies in the SM sample). Thus we conclude that starting with the BGS to establish a $12 \mu \mathrm{m}$ complete sample misses most of the Seyfert 1 galaxies, the sources in the $12 \mu \mathrm{m}$ selected sample that represent an extreme in infrared color. Otherwise the $12 \mu \mathrm{m}$ subsample of the BGS properly describes the brightest galaxies at $12 \mu \mathrm{m}$. The galaxies with extreme values of $S_{v}(12 \mu \mathrm{m}) / S_{v}(60 \mu \mathrm{m})$ are quite rare, and can be ignored for the purpose of measuring the luminosity density of the local Universe at $12 \mu \mathrm{m}$.

A direct comparison of the $12 \mu \mathrm{m}$ subsample with the SM sample after accounting for an apparent difference in the calibration used between the two samples shows that one galaxy (NGC 2841) that should have been included in a complete $12 \mu \mathrm{m}$ flux density limited subsample, was not included in the BGS subsample. NGC 2841 has a ratio of $S_{v}(12 \mu \mathrm{m}) / S_{v}(60 \mu \mathrm{m})=0.20$, as large as the largest found in the $12 \mu \mathrm{m}$ BG subsample. The inverse comparison of the BGS $12 \mu \mathrm{m}$ subsample with the SM sample is not as good. Fifteen galaxies of the 122 galaxies in the BG $12 \mu \mathrm{m}$ subsample that should have been found in the SM sample were not. The SM sample was selected from sources in the IRAS point-source catalog, and this might have lead to the omission of galaxies that were substantially extended at 12 $\mu \mathrm{m}$.

The distribution of the ratio of $S_{v}(25 \mu \mathrm{m}) / S_{v}(60 \mu \mathrm{m})$ in the BGS $25 \mu \mathrm{m}$ subsample can be compared with the IRAS warm galaxy sample (Sanders et al. 1988), selected to have $S_{v}(25 \mu \mathrm{m}) / S_{v}(60 \mu \mathrm{m})>0.2$. In the warm galaxy sample six of 87 objects have values of $S_{v}(25 \mu \mathrm{m}) / S_{v}(60 \mu \mathrm{m})>0.5$, the largest value found in the $25 \mu \mathrm{m}$ subsample. Since the color selection in the warm galaxy sample corresponds to only the $20 \%$ of the galaxies with the largest values of $S_{v}(25 \mu \mathrm{m}) / S_{v}(60 \mu \mathrm{m})$ in the 25 $\mu \mathrm{m}$ sample, we conclude that no more than $1-2 \%$ of galaxies in a $25 \mu \mathrm{m}$ sample would have been missed by our selection procedure.

The sample of UGC galaxies detected in the IRAS survey (Bothun et al. 1989) shows less than 2\% of the galaxies with $S_{v}(60 \mu \mathrm{m}) / S_{v}(100 \mu \mathrm{m})<0.2$, the lowest value found in the $100 \mu \mathrm{m}$ selected survey. Again this suggests there is only a very small fraction of galaxies with such cold color temperatures between 60 and $100 \mu \mathrm{m}$.

These comparisons with other large samples of galaxies demonstrate that galaxies with colors at the extremes which are missed in the BG subsamples are quite rare, and the in- 
completeness of the wavelength-selected BGS subsamples caused by the omission of these galaxies is negligible for the purposes of understanding the general properties of galaxies and for calculating the luminosity functions at these wave- lengths. On the other hand it must be noted that these samples cannot be used for making proper assessments of galaxies with extreme properties based on IRAS colors.
Aaronson, M. 1978, ApJL, 221, L103

Allamandola, L. J., Tielens, A. G. G. M., and Barker, J. R. 1989, ApJS, 71, 733

Bothun, G., Lonsdale, C. J., and Rice, W. L. 1989, ApJ, 341, 129

Boulanger, F., Beichman, C. A., Desert, F. X., Helou, G., and Perault, M. 1988, ApJ, 330, 964

Devereux, N. A., and Young, J. S. 1990, ApJ, 359, 42

Felton, J. E. 1977, AJ, 82, 861

Jura, M., Kim, D. W., Knapp, G. R., and Guhathakurta, P. 1987, ApJ, 312, L11

Lawrence, A., Walker, D., Rowan-Robinson, M., Leech K. J., and Penston, M. V. 1986, MNRAS, 219, 687

Malkan, M. 1990, private communication

Perault, M., Boulanger, F., Puget, J. L., and Falgarone, E. 1990, ApJ (submitted)

Puget, J. L. 1990, private communication

Puget, J. L., and Leger, A. 1989, ARA\&A, 27, 161

Rieke, G. H., and Lebofsky, M. J. 1986, ApJ, 304, 326

Rowan-Robinson, M., Helou, G., and Walker, D. 1987, MNRAS 227,589.

\section{REFERENCES}

Sanders, D. B., Soifer, B. T., Elias, J. H., Neugebauer, G., and Matthews, K. 1988, ApJL, 328, L35

Saunders, W., Rowan-Robinson, M., Lawrence, A., Efstathiou, G., Kaiser, N., Ellis, R. S., and Frenk, C. S. 1990, MNRAS, 242, 318

Smith, B. J., Kleinmann, S. G., Huchra, J. P., and Low, F. J. 1987, ApJ, 318, 161

Schmidt, M. 1968 ApJ, 151, 393

Soifer, B. T., Rice, W. L., Mould, J. R., Gillett, F. C., Rowan-Robinson, M., and Habing, H. J. 1986, ApJ, 304, 651

Soifer, B. T., Sanders, D. B., Neugebauer, G., Danielson, G. E., Lonsdale, C. J., Madore, B. F., and Persson, S. E. 1986, ApJL, 303, L41

Soifer, B. T., Houck, J. R., and Neugebauer, G. 1987, ARA\&A 25, 187

Soifer, B. T., Sanders, D. B., Madore, B. F., Neugebauer, G., Danielson, G. E., Elias, J. H., Lonsdale, C. J., and Rice, W. L. 1987, ApJ, 320, 238 (Paper I)

Soifer, B. T., Boehmer, L., Neugebauer, G., and Sanders, D. B. 1989, AJ, 98, 766 (Paper II)

Spinoglio, L., and Malkan, M. A. 1989, ApJ, 342, 83 (SM) 